\title{
Fünf Jahre SGB II - Versuch einer Bilanz
}

Ist Hartz IV, wie vielfach behauptet, tatsächlich Armut per Gesetz - und damit ein eklatanter Bruch der deutschen Sozialstaatstradition? Und was wäre dann das grundsätzlich Neue am neuen System der Grundsicherung für erwerbsfähige Hilfebedürftige? Oder bewegt sich das neue System weitgehend in den alten Gleisen seiner Vorgänger? Oder haben wir es vielleicht mit einem allmählichen Wandlungsprozess zu tun? Der Beitrag zeigt: In einem längeren historischen Betrachtungszeitraum und bei einer Zusammenschau der bislang vorliegenden empirischen Befunde werden sowohl überraschende Kontinuitäten sichtbar wie auch langsame Wandlungsprozesse, konfligierende Konzepte von Gerechtigkeit und ein sich wandelndes Bild vom Hilfebedürftigen. ${ }^{1}$

\section{1 \\ Einleitung}

Seit Januar 2005 besteht in Deutschland ein einheitliches Grundsicherungssystem für erwerbsfähige Hilfebedürftige - ein Kernstück der Arbeitsmarkt- und Sozialreformen der damaligen rot-grünen Bundesregierung, die sich von angelsächsischen Vorbildern inspirieren ließ. Vorbereitet von der mit Wissenschaftlern, Politikern und Managern prominent besetzten und dem ehemaligen VW-Manager Peter Hartz geleiteten gleichnamigen Kommission wurde das neue Sozialgesetzbuch (SGB) II nach teils erbitterten öffentlichen Debatten eingeführt. Dies bedeutete nicht nur die administrative Zusammenlegung und Vereinheitlichung der beiden vormals getrennten Unterstützungssysteme der Arbeitslosenund der Sozialhilfe, ${ }^{2}$ sondern es drückte auch, zumindest in der politischen Rhetorik und im Gesetzestext, eine stark veränderte Ausrichtung der dahinterstehenden Ziele und Motive aus. Stand in der Praxis der deutschen Sozialhilfe bis dato vor allem die Versorgung der Betroffenen zur Kompensation materieller Notlagen im Sinne einer Armutsabwehr im Vordergrund, so geht es nunmehr, wie in den Workfare- und Activation-Konzepten anderer Wohlfahrtsstaaten, vorrangig um die möglichst rasche Beendigung der Armutslage durch die Vermittlung einer Existenz sichernden Erwerbsarbeit, was durch verschiedene Aktivierungsmaßnahmen erreicht werden soll.

$\mathrm{Zu}$ fragen ist: Ist das neue System aus Grundsicherung und Aktivierung ein tief greifender Wandel der Armutsbekämpfung? Steckt dahinter gar ein sozialpolitischer Paradigmenwechsel, nicht nur auf rhetorischer Ebene, sondern auch in der Praxis?
Nahezu fünf Jahre im neuen System Hartz IV, unter Beobachtung der kritischen Öffentlichkeit und der begleitenden Evaluationsforschung, sind Anlass, eine Bewertung vorzunehmen. Es soll gezeigt werden, dass das geänderte Menschenbild, Entbiografisierung und Entdifferenzierung sowie der Konflikt zweier Gerechtigkeitskonzepte die wesentlichen Charakteristika eines längerfristigen Entwicklungsprozesses sind, in dem die Hartz-IV-Reform einen wichtigen Eckpunkt bildet. Die folgenden Ausführungen verstehen sich dabei als Deutungsvorschlag, nicht als erschöpfende Bestandsaufnahme vergangener und gegenwärtiger Armutsbekämpfung und Sozialpolitik. Sie sollen eine fundierte Diskussion eröffnen, nicht abschließen.

Abschnitt 2 befasst sich mit grundlegenden Ambivalenzen und Spannungsverhältnissen der Armutsbekämpfung in Deutschland. Länger angelegte Wandlungsprozesse der Sozialpolitik sind Gegenstand des Abschnitts 3. Anschließend erfolgt eine kurze empirische Bilanz der Hartz-IV-Reform (Abschnitt 4). Wie neu der mit Hartz IV verbundene Aktivierungsansatz wirklich ist, wird im Abschnitt 5 erörtert. Das Schlusskapitel bietet den Versuch einer Synthese (Abschnitt 6).

\section{Ambivalenzen der Armutsbekämpfung in Deutschland}

Es ist eine Grundtatsache des sozialen Lebens, dass manche Menschen ihren Lebensunterhalt nicht alleine bestreiten können. In bestimmten biografischen Phasen ist dies sogar normal, etwa in der Kindheit, im Alter oder bei Krankheit. Dass Menschen der unmittelbaren Umgebung, sei es
Familie, Stamm, Dorf, Kultusgemeinde oder andere sozialen Gruppen, in solchen Fällen Unterstützung und Hilfe leisten, zählt gleichfalls zu den Grundstrukturen menschlicher Gemeinschaftsbildung. Doch manchmal reichen diese primären Unterstützungsbeziehungen nicht aus.

\subsection{ENTSTEHUNG VON ARMUTS- UND SOZIALPOLITIK}

Dies war und ist immer noch der Fall bei Kriegen, Naturkatastrophen, wirtschaftlichen oder gesellschaftlichen Krisen und Zusammenbrüchen - oder in Wandlungsprozessen des menschlichen Zusammenlebens, bei denen sich Strukturen, Formen und Funktion primärer Gruppen oder Gemeinschaften drastisch verändern. Ein klassisches Beispiel für Letzteres ist der Übergang von agrarischen, feudal oder

\footnotetext{
1 Dieser Aufsatz ist die stark überarbeitete und erweiterte Fassung eines Beitrags zum ersten Bericht des $I A B$ an das Bundesministerium für Arbeit und Soziales (BMAS) im Rahmen der Forschung nach $\S 55$ SGB II (Koch et al. 2009). Der Verfasser dankt Jürgen Kühl, Claus Offe, Philipp Ramos-Lobato, Gabriele Wollnik und zwei anonymen Gutachtern der WSI-Mitteilungen für hilfreiche Kommentare.

2 Streng genommen wurden die beiden Systeme nur für erwerbsfähige Hilfebedürftige zusammengelegt; die Sozialhilfe existiert im SGB XII weiter. Der weitaus größte Teil der vormaligen Sozialhilfeempfänger ist jedoch in den Rechtskreis SGB II gewechselt, sodass von einer faktischen Vereinheitlichung zu sprechen ist.
}

Markus Promberger, Dr. phil., Soziologe, ist Leiter des Forschungsbereichs "Erwerbslosigkeit und Teilhabe" am Institut für Arbeitsmarkt- und Berufsforschung (IAB) in Nürnberg. Arbeitsschwerpunkte: Armuts-, Arbeits- und Arbeitsmarktforschung. e-mail: markus.promberger@iab.de 
im größeren Dorfverband eingebundenen Großfamilien zu urbanen Klein- und Kernfamilien, mit gleichzeitig abnehmender wirtschaftlicher Binnenleistung, abnehmender Personenzahl und zunehmenden wirtschaftlichen Außenbeziehungen. Als Reaktion darauf kann z. B. die Bildung und Ausdifferenzierung zünftiger Selbsthilfeeinrichtungen städtischer Handwerker gelten; als - über Zwischenstufen - anschließende weitere Entwicklung die Entstehung des modernen Sozialstaats. Ein anderes Beispiel ist der quantitative Bedeutungsgewinn von unvollständigen oder Patchworkfamilien mit zum Teil nur noch einem beständig zugehörigen Erwachsenen. Fest steht jedenfalls, dass sich bereits seit der Antike immer wieder gesetzliche Regelungen und öffentliche Unterstützungseinrichtungen nachweisen lassen, die hilfebedürftigen Menschen beispringen, sofern deren Familien oder Bezugsgruppen nicht ausreichend dazu imstande sind (Frerich/ Frey 1996a; Prell 1997). Sekundäre Institutionen treten neben die primären Institutionen.

Das Massenelend der Industrialisierung und die genuinen Existenzrisiken der Lohnarbeit schließlich stießen politische Prozesse an, Wohlfahrtsstaatlichkeit zu entwickeln, in der breite und entfaltete ,sekundäre Institutionen“ der Sozialpolitik auch die Menschen in Armutslagen unterstützen. Begrifflich und politisch lässt sich dabei ein Übergang von der Armenfrage des Mittelalters über die Soziale Frage des 19. und frühen 20. Jahrhunderts bis zur entwickelten wohlfahrtsstaatlichen Sozialpolitik seit etwa 1927-1970 aufzeigen, die Armut - bis zu Heiner Geißlers „, neuer Armut" - mehr und mehr verschiedenen Rand- und Problemgruppen zuschreibt, während vormals zentrale Armutsrisiken (Alter, Krankheit, Arbeitslosigkeit) nunmehr durch die flankierenden Institutionen des Normalarbeitsverhältnisses abgedeckt sind.

\subsection{ARMUTSPOLITIK ZWISCHEN AUSGRENZUNG, KONTROLLE UND UNTERSTÜTZUNG}

Schon seit Jahrhunderten ist dabei ein charakteristischer Zwiespalt des gesellschaftlichen Umgangs mit Armut zu beobachten (Friedeburg, v. 2002): Armut ruft einerseits Solidarität, Mitleid und Unterstützung hervor, andererseits auch Ablehnung, Irritation und Ausgrenzung, insbesondere wenn sie mit abweichenden Lebensweisen verbunden ist - was von religiöser oder ethnischer Abweichung bis zu Abweichungen von standardisierten Rollenmustern (z. B. Hausfrau, Arbeiter, Staatsbürger, Sesshaftigkeit) reichen kann. Auf diesem Zwiespalt fußt die bekannte Unterscheidung von ,deserving" und „undeserving poor". Nur so erklärt sich auch, dass Armutsbekämpfung seit ihren Anfängen bis heute zwei Gesichter hat: Das eine ist Versorgung und Unterstützung, das andere ist die „armenpolizeiliche" Perspektive von Ordnung, Kontrolle, Zwang und Ausgrenzung, die von Bettelverboten über Zwangsarbeit bis zu Armenhäusern und Deportation reichen konnte.

Eine zunächst umstrittene, für die weitere Entwicklung jedoch folgenreiche Zäsur war das Grundsatzurteil des Bundesverwaltungsgerichts von 1954, das mit seiner Anerkennung des Sozialstaatsgebots im Grundgesetz und des subjektiven Anspruchs auf Unterstützung letztlich die in anderen westlichen Demokratien dominante Vorstellung von Sozialleistungen als Bürgerrecht (Marshall 1992) auch in Deutschland durchsetzte (Willing 2005a, S. 596; Frerich/Frey 1996b, S. 124). Dieser Grundrechtsgedanke stand später Pate für das Bundessozialhilfegesetz von 1961. Dadurch wurde die „armenpolizeiliche“ Seite der Sozialpolitik allmählich weitgehend auf Bedürftigkeitsprüfungen, Mitwirkungspflichten und Bekämpfung des Leistungsmissbrauchs reduziert. Gleichwohl gibt es immer noch gesellschaftliche Ausgrenzungsdiskurse - wie die Unterschichtdiskussion des Sommers 2007 gezeigt hat. Die neuesten kritischen Chiffren der politischsozialen Sprache hierfür sind Exklusion (z. B. Kronauer 2002) und Prekarität (z. B. Castel 2000). Beiden gemeinsam ist die Diagnose, dass die Integrationskraft der Arbeitsgesellschaft seit ihrer Hochphase der 1950er und 1960er Jahre abgenommen hat; an ihren Rändern haben sich prekäre Erwerbsverhältnisse und ökonomisch instabile Lebenslagen entwickelt, zu denen mehr oder weniger wohlfahrtsstaatlich versorgte Arbeitslosigkeit und minderprivilegierte Erwerbsformen sowie deren Mischformen (working poor, Unterbeschäftigung) gehören. Im schlimmsten Falle sind Betroffene fast vollständig von Ressourcen, Lebensräumen und Alltagspraktiken der Mehrheitsgesellschaft ausgeschlossen.

In der Geschichte der Sozialpolitik sind wir somit bei allen Entwicklungen in Ge- sellschaft, Wirtschaft und Politik mit erstaunlichen strukturellen Kontinuitäten konfrontiert: Das Neben- und Miteinander von Versorgung und Kontrolle (Marx 1984; Foucault 1975), die Differenzierung der Armen zwischen "deserving “ und „undeserving poor" (Lis/Soly 1979), die Frage, ob die Armen zur Gesellschaft gehören oder nicht (Friedeburg, v. 2002) markieren die Janusköpfigkeit der Armutspolitik mit Ausgrenzung, Einschließung, Versorgung und Kontrolle, Unterstützung und Gegenleistungserwartung immer noch, auch wenn die Armutspolitik sich mittlerweile einigermaßen konsistent an den staatsbürgerlichen Grundrechten orientiert.

Kontinuität zeigt sich auch hinsichtlich des andaurnden Streits über das angemessene Niveau materieller Unterstützungsleistungen. Hier geht es um das Spannungsfeld zwischen „objektiven“ Grundbedürfnissen (Standardwarenkorb oder soziokulturelles Existenzminimum), dem sogenannten Lohnabstandsgebot und dem regionalen und lebenslagenabhängigen Differenzierungsbedarf, was zum periodischen Wechsel zwischen Forderungen nach Anhebung und Absenkung der materiellen Unterstützungsleistungen führt. Schwerpunkt der jüngeren Debatte ist die Angemessenheit der Regelleistung insbesondere für Familien; eine Frucht dieser Diskussion ist die Anhebung des Sozialgeldes für Kinder im Schulalter ab Juli 2009.

Auch die Grundstruktur der Subsidiaritätskette ist seit der frühen Neuzeit nahezu unverändert geblieben: Der Einstand für Hilfebedürftige beginnt im engeren und weiteren Familienzusammenhang, dann folgt ein Nebeneinander von Berufs-, Standes- oder Kultusgemeinschaften, teils von vorangegangenen Eigenbeiträgen abhängig, teils auf karitativer Grundlage, dann die Dorfgemeinschaft, Stadt oder Kommune, sodann - und historisch spät besondere staatliche Einrichtungen und überörtliche karitative Einrichtungen. Nur: Die Zuständigkeitskette ist institutionell und räumlich lückenloser geworden, überlappende Zuständigkeiten sind seltener, Gewichtsverlagerungen zwischen den Akteuren haben stattgefunden, sodass die Nichtinanspruchnahme zustehender Sozialleistungen lediglich noch von Informationsdefiziten, Scham, Ausgrenzungserwartungen oder geringem Zusatznutzen abhängt (Becker/Hauser 2005).

Relativ ähnlich geblieben ist in the long run auch die funktionelle Differenzierung 
der Versorgung Hilfebedürftiger nach der Art des Risikos. Prüfungen hinsichtlich ökonomischer Bedürftigkeit und moralischer Hilfewürdigkeit sind seit dem Mittelalter üblich. Hilfebedürftige Kranke motivierten zur Schaffung separater Einrichtungen - Krankenhäuser, Krankenspitäler -, wobei in neuerer Zeit semantische Verschiebungen von der Krankheit zur Erwerbsunfähigkeit konstatiert werden müssen. Auch Krieg erzeugt in der Regel separate Einrichtungen für Kriegsteilnehmer und Betroffene - von der Veteranenversorgung des revolutionären Frankreichs bis zu den Kriegs- und Hinterbliebenenrenten der frühen Bundesrepublik. Hilfebedürftigkeit im „dritten Lebensalter“ ist und war meistens in eigenen Systemen geregelt; gleiches gilt auch für Kinder, insbesondere wenn deren familiärer Unterstützungskontext nicht vorhanden ist oder versagt.

Hilfebedürftigkeit im erwerbsfähigen Alter und bei - wie auch immer definierter - Erwerbsfähigkeit erfordert ebenfalls ein eigenes System, dem hier unser Hauptaugenmerk gilt. Es entstand in der Weimarer Republik in Abgrenzung von der übrigen Armenfürsorge und als Ergänzung der teils kommunal geförderten Selbsthilfeund Versicherungssysteme, die 1927 in die reichsweite Arbeitslosenversicherung des AVAVG einmündeten. Dieses Regelwerk blieb, vielfach geändert, rund 40 Jahre in Kraft und wurde 1969 vom Arbeitsförderungsgesetz (AFG) abgelöst, welches wiederum 1997 zum Sozialgesetzbuch III umgebaut wurde. Bei diesem Umbau blieb die 1926 als sogenannte Krisenfürsorge eingeführte Arbeitslosenhilfe zunächst im Grundsatz bestehen, bis zur Einführung des Sozialgesetzbuchs II. Die Doppelgleisigkeit der Armutsbekämpfung aus allgemeiner Fürsorge/Sozialhilfe und Erwerbslosenfürsorge/Arbeitslosenhilfe bildete somit eine annähernd 80 Jahre währende feste Größe deutscher Sozialpolitik.

\section{3 \\ Wandlungsprozesse der Armutspolitik}

Die deutsche Sozialpolitik war jedoch auch von vielfältigen allmählichen Veränderungsprozessen geprägt - und zwar schon vor den Reformen 2005. Der Bedeutungsverlust direkter, interpersonaler klassenübergreifender Schutzverhältnisse (Patri- ziat, Klientelismus, Patronage) und der betrieblichen Sozialpolitik wie auch das erfolgreiche Vordringen des Grundrechtsgedankens in die Armutsbekämpfung sind bekannt. Ebenso hat sich die Rolle der Mildtätigkeit geändert. Es gibt sie nach wie vor, sie hat nur eine Abstraktionsbewegung durchlaufen. Man spendet meist (im Abendland) nicht mehr dem persönlich bekannten Armen, sondern Organisationen, die sich teils ehrenamtlich, teils professionell um Armut kümmern oder andere wohltätige Aufgaben versehen.

\subsection{VEREINHEITLICHUNG UND RÜCKBAU VON STATUSDIFFERENZEN...}

Hundert Jahre wohlfahrtsstaatlicher Entwicklung in Deutschland zeigen überdies eine deutliche Vereinheitlichungsbewegung der öffentlichen Armutsbekämpfung. Dies bedeutete einen Rückbau vor allem statusbezogener Differenzierungen. So unterschied die Fürsorgereform von 1924 (wie ihre Vorgänger) neben anderen Systemen etwa noch Erwerbslosenfürsorge, Armenfürsorge, Kriegsbeschädigte, Sozial- und Kleinrentner, Schwerbeschädigte, Wöchnerinnen und Wanderer - und wies einigen von ihnen die sogenannte "gehobene Fürsorge“ $z u$, bei der die „früheren Lebensverhältnisse" auch in Art und Umfang der Unterstützungsleistungen zu berücksichtigen waren. Ähnliche gruppen- bzw. statusbezogene Differenzierungen wurden noch in den 1950er Jahren diskutiert und teilweise praktiziert, doch Zug um Zug abgebaut sieht man von den nach wie vor abgetrennten Regelkreisen für nicht erwerbsfähige Menschen ab. Der vorläufig letzte Schritt dieser Vereinheitlichung, der erst mit dem SGB II vollzogen wurde, ist der Wegfall der Arbeitslosenhilfe, die anteilsmäßig am vormaligen Arbeitsentgelt (53\% bzw. $57 \%$ ) orientiert war, nicht jedoch an der Aufrechterhaltung des Lebensstandards. Damit gibt es nun kein an der vorangegangenen Erwerbsbiografie orientiertes Unterstützungssystem für erwerbsfähige Hilfebedürftige mehr. Dies ist nicht nur ein Problem der z. T. unterschiedlichen Höhe der Transferzahlungen, sondern auch des Wegfalls der sozialen Anerkennung der erwerbsbiografischen Leistung. Für vormalige Sozialhilfeempfänger ist der Monatsbetrag des Arbeitslosengeld II zwar nun teilweise höher, doch sind die Einmalzahlungen der Sozialhilfe im SGB II rech- nerisch in den Pauschalbetrag eingeschlossen, was nach Meinung vieler Experten einem faktischen Wegfall gleichkommt: „Wenn eine Familie mit kleinen Kindern in Hartz IV eine neue Waschmaschine braucht, können wir heute nur noch eine Spende vermitteln" beschreibt eine verantwortliche Sozialamtsmitarbeiterin die Situation. $^{3}$

Jenseits der Härte des Schicksals, die dies im Einzelfall bedeutet, lässt sich diese Entwicklung verstehen als Meilenstein einer fortschreitenden Entdifferenzierung oder Egalisierung der Armutsbekämpfung, die keine Unterscheidungen innerhalb der Hilfebedürftigen vornimmt, zumindest sofern sie erwerbsfähig sind. Die „Versorgungsklasse“ (Max Weber) der Armen wird weiter homogenisiert.

\section{2 ...UND GEGENBEWEGUNGEN}

Doch ist fraglich, ob diese Egalisierung tatsächlich den Endpunkt der Entwicklung darstellt. Denn auch heute wieder, fast fünf Jahre nach der Einführung des SGB II, werden situationsbezogene Zuschläge diskutiert, etwa für Alleinerziehende oder Familien mit Kindern. Zudem stellt sich die Frage, ob die Egalisierungsbewegung auf der gesetzlichen Regelungsebene nicht immer wieder durch eine möglichst situationsund problemadäquate Handhabungspraxis vor Ort aus guten Gründen konterkariert wird - denn auch die Heterogenität der Armutspopulation und ihrer Lebenslagen ist eine unbestreitbare und auch von der historischen Forschung (z. B. Friedeburg, v. 2002; Willing 2005a) vielfach dargestellte Konstante. Sie äußert sich gegenwärtig beispielsweise darin, dass hinsichtlich zu treffender Maßnahmen immer wieder zwischen „erwerbsnahen“ und „erwerbsfernen "Hilfebedürftigen unterschieden wird - und damit eine Trennungslinie gezogen wird, die wenigstens teilweise mit der vorherigen Unterscheidung zwischen Arbeitslosen- und Sozialhilfe kongruent ist. Auch im Hinblick auf biografisch erworbene oder legitimierbare Status-, Versorgungs- oder Anerkennungsansprüche ist die Diskussion noch lange nicht beendet, wie auch die 2008 erfolgte Wiederverlängerung des Arbeitslosengeldes I ge-

3 Das Zitat entstammt einem im Rahmen des IAB-
Projektes "Leben, Arbeit und soziale Sicherheit" geführten Interview (Promberger 2007). 
zeigt hat. In jedem Falle ist das Verhältnis der solidarisch-egalitären Gerechtigkeitsansprüche zu den biografisch erworbenen Gerechtigkeitsansprüchen nach wie vor, und vermutlich auch künftig, von erheblicher Bedeutung für die Sozialpolitik in Deutschland.

\subsection{ZWISCHENFAZIT}

Kontinuitäten - auch im Sinne fortbestehender Spannungen - und langsamer Wandel bestimmen die Entwicklung der Sozialpolitik, insbesondere der Armutsbekämpfung auch nach 2005. Dennoch waren die Hartz-Kommission und das SGB II mit dem Anspruch eines deutlichen Politikwechsels angetreten, der durch veränderte wirtschaftspolitische Rahmenbedingungen ausgelöst worden war. Denn folgt man Robert Castel (2000), Ulrich Beck (1999) und anderen Zeitdiagnostikern, so verlieren traditionelle industrielle Arbeitsarrangements an Inklusionskraft. Entsprechend verlieren auch die darauf aufbauenden und deren Risiken kompensierenden Institutionen des Wohlfahrtsstaats an Leistungsfähigkeit und Bedeutung. Ein wirtschaftspolitischer Paradigmenwechsel zu Monetarismus und (Neo-)Neoklassik, veränderte Vorstellungen von der Rolle des Staates in der Wirtschaft, aber auch die Schulweisheit, dass man nicht dauerhaft mehr ausgeben könne als man einnimmt, machten bei fortdauernder ,struktureller“, teils auch durch neue arbeitssparende Produktionstechnologien und Produktionsverlagerung in andere Weltregionen bedingter Arbeitslosigkeit. ${ }^{4}$ Haushaltskonsolidierung und Geldwertstabilität zum Leitmotiv einer nachkeynesianischen Wirtschaftspolitik - auch wenn einzuwenden bleibt, dass Deutschland weder jemals lupenreinen Keynesianismus praktiziert hat noch je eine vollständige arbeitspolitische Wende im Stil von Thatcher und Reagan vollzogen hat. Trotzdem sind deutliche Einschnitte in den Leistungen von Arbeitslosenversicherung und Sozialhilfe seit etwa 1980 Ausdruck dieses Strategiewechsels. In den 1990er Jahren rückte die schnelle Beendigung von Sozialleistungsbezug durch die Wiederaufnahme einer Existenz sichernden Erwerbsarbeit zum Leitthema vor allem der Arbeitsmarktpolitik auf. Flankierend für diesen Mix aus Leistungseinschnitten und verstärkten Bemühungen, ja bisweilen Zumutungen der Reintegration in Erwerbsarbeit wirkten vor allem folgende Faktoren: Die fiskalischen Lasten der Wiedervereinigung und der unbewältigten Strukturkrise des Arbeitsmarktes, die langjährige, teils selektiv geführte kritische Debatte um die Arbeitsbeschaffungsmaßnahmen (Schmid/Oschmiansky 2005, S. 270ff.), die wissenschaftlich-politischen Diskussionen um die Armutsfalle (vgl. kritisch Gebauer et al. 2002) und die obsolet gewordene Systemkonkurrenz mit dem realen Sozialismus, die nach Ansicht von Zeithistorikern (Rudloff 1998) einen beträchtlichen Teil zur Expansion des bundesdeutschen Wohlfahrtsstaates beigetragen hatte. Hierdurch wich die vormals vordergründig dominante Programmatik von Versorgung und Befähigung zu eigenständig-selbstbestimmtem Leben ${ }^{5}$ dem Ansatz einer möglichst effektiven Arbeitsmarktreintegration, in der trügerischen Hoffnung, dass dies effizienter und billiger wäre. Der Umbau des Sozialstaats stellt in dieser Perspektive eine Konsequenz des sozioökonomischen Wandels dar. Mit dem SGB II sollte der Übergang vom versorgenden (Hradil 2006) oder fürsorgenden zum aktivierenden Sozialstaat (Dingeldey 2006) angetreten werden; eine programmatische Abkehr von den als nicht länger tragbar empfundenen Gewohnheiten des herkömmlichen Wohlfahrtsstaats wurde angestrebt. Was hat sich davon empirisch in der SGB-II-Reform niedergeschlagen?

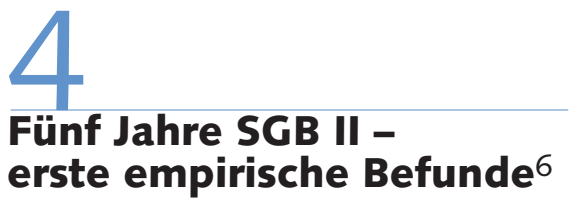

Überraschend war der hohe Zuwachs an Hilfebeziehern in den ersten drei Monaten nach der Systemumstellung am 1.1.2005. Selbst nach der Saldierung der Übergänge aus den alten Systemen der Arbeitslosenund Sozialhilfe und den saisonal erwartbaren Zugängen mit den erwartbaren Abgängen aus dem Hilfebezug durch Fluktuation, durch die Haushaltsveranlagung und die geänderten Freibetragsregelungen blieb ein Zuwachs von rund einer Million (1,027 Mio.) unerwarteten neuen Hilfebedürftigen. Die einzige plausible Erklärung hierfür ist eine verstärkte Mobilisierung von Bedürftigen, die bislang ihnen zustehende Sozialleistungen nicht genutzt hatten, sei es wegen fehlender Information, geringem Zusatznutzen bei kleinen Erwartungsbeträgen oder aus Scham (vgl. zur Dunkelzif- ferdiskussion Becker/Hauser 2005). Allein die Tatsache, dass der Einführung der neuen Grundsicherung monatelange breite öffentliche Kontroversen vorangingen, aber auch das geringere soziale Stigma der Arbeitslosigkeit gegenüber dem Sozialhilfebezug sowie die räumliche, organisatorische und damit soziale Nähe vieler Grundsicherungsträger zum Arbeitsamt mögen zum Abbau von Scham und Informationsdefiziten beigetragen haben. Gleichzeitig hat der geringfügig erhöhte Pauschalbetrag gegenüber den vormaligen Regelsätzen der Sozialhilfe die Inanspruchnahme geringer zustehender Leistungsbeträge rentabler gemacht (Rudolph 2006; Promberger 2008a). Wer also von der Grundsicherungsreform eine finanzielle Entlastung des Sozialstaats erwartet hatte, musste angesichts dieser Tatsachen enttäuscht sein.

Der Gesetzgeber hatte vorgesehen, dass die Betreuung des neuen Systems im Normalfall eigens zu bildenden gemeinsamen Einrichtungen von Arbeitsverwaltung und Kommunen obliegen sollte, den sogenannten SGB-II-Arbeitsgemeinschaften, kurz ARGEn. Daneben sah der $\$ 6$ c SGB II eine Experimentiermöglichkeit für 69 Gebietseinheiten vor, in denen meist kommunale Träger die Betreuung der Hilfebedürftigen übernehmen sollten. Jenseits von parteipolitischen Präferenzen war dies vor allem eine von den kommunalen Spitzenverbänden unterstützte Wunschlösung für manche Kommunen, die den Verlust ihrer Eigenhoheit in der Sozialpolitik befürchteten. Trotz intensiver Analyse sind jedoch bislang keine eindeutigen Vorteile dieser Sonderlösung zu erkennen, vielmehr scheint sich ein leichter Vorteil der ARGEn abzuzeichnen - Ansätze überregionaler Zusammenarbeit und einheitlicher Statistik eingeschlossen (Bundestag 2009).

Erste Analysen (Christoph 2008) belegen klare Unterversorgungslagen, vor allem im Bereich neuer und besserer Kleidung, Möblierung und Ressourcen für kulturelle und soziale Teilhabe. Ob dies ein Effekt der Pauschalierung oder schlichtweg

\footnotetext{
Eine erste zeithistorische Aufarbeitung der "Krise der Arbeitsgesellschaft " bieten Schlemmer/Raithel (2009).

5 \$1 Abs. 2 BSHG von 1961.

6 Den Befunden in diesem Abschnitt liegt, soweit nicht anders erwähnt, eine Vielzahl von Einzelveröffentlichungen aus dem IAB zugrunde; vgl. Achatz et al. (2009) und den digest von Koch et al. (2009).
} 
des niedrigen Regelsatzes ist, ob sich der Effekt durch die Grundsicherungsreform verstärkt hat oder nicht, kann wegen des Fehlens von Längsschnittinformationen noch nicht geklärt werden. Qualitative Befunde des IAB zeigen jedenfalls, dass sich in betroffenen Familien bei längerdauernder Hilfebedürftigkeit ein schleichender Einschränkungsprozess des materiellen Lebensniveaus wie auch der realisierten sozialen Teilhabe vollzieht (Hirseland et al. 2009).

Die „Working Poor“, also Menschen, die zusätzlich zum Arbeitseinkommen noch ergänzende Sozialleistungen beziehen, sind durch die Reform verstärkt ins Blickfeld geraten. Das Gleiche gilt für diejenigen, die zum Arbeitslosengeld I noch Grundsicherungsleistungen erhalten („Aufstocker"). Dahinter eine Wirkung des neuen Systems zu vermuten, wäre falsch, denn ergänzenden Sozialhilfebezug gab es schon vorher. Eine Zunahme ist wegen der lückenhaften Datenlage zum Sozialhilfebezug vor 2005 schwer zu belegen, jedoch wegen der Veränderungen im Tarif- und Lohngefüge (vgl. Ellguth/Kohaut 2008; Bellmann/Gartner 2003) sowie aufgrund des Wachstums prekärer Beschäftigung zu vermuten.

Für viele Beobachter ebenfalls überraschend, aus Sicht der Armutsforschung hingegen altbekannt, ist die Heterogenität der Hilfebezieher - der „erwerbsfähigen Hilfebedürftigen“. Sie bilden keinesfalls eine homogene Gruppe von Exkludierten und Außenseitern, Arbeitsmarktfernen und Langzeitarbeitslosen. Etliche - vor allem die Jüngsten - befinden sich in schulischen und beruflichen Bildungsprozessen. Sie sind wegen der Arbeitslosigkeit eines Familienmitglieds hilfebedürftig geworden. Manche betreuen kleine Kinder, teils ohne Partner, wiederum andere sind krank oder behindert; sie sind zwar nominell drei Stunden pro Tag erwerbsfähig, finden jedoch bei ihrer stark eingeschränkten Kondition faktisch keinen Arbeitsplatz. Wiederum andere sind keineswegs arbeitsmarktfern, sehnen sich nach Arbeit, haben Minijobs, organisieren ihren Alltag vernünftig, manche von ihnen wirken gar, als könnten sie sich nach dem Gespräch mit ihrem Betreuer bei der ARGE sofort zur Spätschicht im benachbarten Waschmaschinenwerk melden - doch das hat eben vor drei Jahren geschlossen.

Altbekannte Befunde aus der Armutsforschung begegnen uns ohnehin auch bei der Analyse des neuen Systems - etwa der Zusammenhang von (persönlicher oder familialer) Arbeitslosigkeit und Armut, das Risiko der Altersarmut nach Arbeitslosigkeit, die schwierigen Einstiege Jugendlicher und junger Erwachsener aus Leistungsbezieherhaushalten in Berufsausbildung und Erwerbsleben (Achatz et al. 2009). Auch die erhöhten Arbeitsmarktrisiken von Menschen mit schlechter schulischer Ausbildung und fehlendem Berufsabschluss, die sich teilweise von Eltern auf Kinder „,vererben“, sind bekannt; in der Forschung zu Hartz IV wird man von Neuem darauf aufmerksam, angesichts des überproportionalen Anteils von „Bildungsverlierern“ unter den Grundsicherungsempfängern. Gleiches gilt für Konsumeinschränkungen und die negative Erfahrung, vor allem von jungen Erwachsenen und Familien mit Kindern (Bosch 2009), dass man an den üblichen und sozial erwarteten Aktivitäten um Schule und Freizeit nicht teilhaben kann (Achatz et al. 2009).

Weitgehend neu ist das mit der HartzIV-Reform und ihren Vorläufern (z. B. „Arbeitsamt 2000“) verbundene Interesse an Betreuung und Aktivierung. Untersuchungen zum Vermittlerhandeln fördern - neben den Einführungs- und Umstellungsschwierigkeiten des neuen Systems (Bartelheimer 2008; Dornette et al. 2008) - regelmäßig erhöhten, auch von den Vermittlern selbst konstatierten Bedarf an professioneller Diagnose- und Betreuungskompetenz jenseits starrer Vorschriften und „naturwüchsiger Pädagogiken“ zutage (LudwigMayerhofer et al. 2009). Professionalisierung scheint das Gebot der Stunde. Die Betreuten selbst reagieren auf die Aktivitäten ihrer Vermittler höchst different, keineswegs werden alle Maßnahmeangebote als echte Chance zur Reintegration in den Arbeitsmarkt wahrgenommen. Das Spektrum der individuellen Deutungsmuster reicht von der empfundenen Zumutung über das Verständnis, dass man angesichts der Grundsicherungsleistung zu einer Gegenleistung verpflichtet sei, bis zur Wahrnehmung von Maßnahmen als Quasi-Arbeitsverhältnis. Nur ein kleinerer Teil der Betroffenen reagiert so, wie es die Mitwirkung an der Aktivierungsidee erfordern würde (Wenzel 2008).

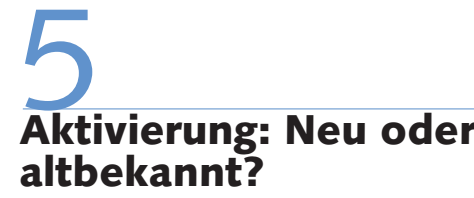

Aktivierung im Sinne von Arbeitsmarktintegration war der Anspruch, mit dem das SGB II angetreten war. Zunächst war schnell festzustellen, dass unter der Oberfläche der Aktivierungsrhetorik nach wie vor die Grundversorgung Hilfebedürftiger die Priorität in der alltäglichen Praxis der neu geschaffenen administrativen Organe des SGB II genoss. Auch die neue arbeitsmarktpolitische Maßnahme der Ein-EuroJobs (AGH), vordergründig auf schnelle Arbeitsmarktintegration und die dazu nötige Beschäftigungsfähigkeit ausgerichtet, wurde oft anders eingesetzt und erwies sich als tauglich und nützlich für soziale Integration, wo infolge der Arbeitslosigkeit die stillschweigenden Integrationsfunktionen marktvermittelter Erwerbsarbeit zeitweise oder dauerhaft fehlten (Promberger 2008b). Die SGB-II-Arbeitsgelegenheiten ähneln ohnehin den Arbeitsgelegenheiten des BSHG (\$\$ 18-20) aufs Haar, es scheint nur, dass Letztere lokal und zeitlich in höchst unterschiedlichem, meist begrenztem Maße zum Einsatz gekommen sind. Auch Familienhilfen, Kinderbetreuungsangebote und psychologische Unterstützung dürften nicht unbedingt Menschen schnell in Jobs bringen, wohl aber psychisch und sozial stabilisieren - auch wenn dazu noch keine empirischen Ergebnisse vorliegen.

Andererseits kam es zu einer beträchtlichen Intensivierung der Arbeitsvermittlungsbemühungen für Langzeitarbeitslose und vormalige Sozialhilfeempfänger, wohl auch zu einer Ausweitung von Sanktionen, aber ebenso zu einer gewissen Erweiterung des Vermögensschutzes, zumindest gegenüber der früheren Sozialhilfe. So sind das eigene Kraftfahrzeug, private Altersvorsorgevermögen und selbst genutztes Wohneigentum innerhalb gewisser Grenzen vom pflichtgemäßen Vermögensabbau im Rahmen des SGB II ausgenommen.

Aktivierung ist also wirklich nicht neu - nur das Wort ist es. So finden sich Aktivierungselemente, wie man sie heute nennen würde, beispielsweise bereits in der Armengesetzgebung des 17. Jahrhunderts in England und Deutschland (vgl. Marx 1984; Lis/Soly 1979; Frerich/Frey 1996a; Sachße/ Tennstedt 1980). Bei allen historischen Differenzen ist diesen Regelungen die Mög- 
lichkeit gemeinsam, Arbeitslose und Arme zu öffentlichen Arbeiten heranziehen zu können. Die faktische Nutzung dieser rechtlichen Optionen unterliegt jedoch erheblichen, seit der industriellen Revolution stark konjunkturell und politisch bedingten Diskontinuitäten. Phasen exzessiver Nutzung, etwa in der Demobilmachung nach 1918, der Weltwirtschaftskrise von 1929 oder in den 1980er Jahren, wechseln sich mit Phasen ausgesprochen geringer Nutzung ab. Es scheint auch, dass nicht alle derartigen Arbeitspflichten mit Zwangsarbeit oder gefängnisähnlichen Armenhäusern gleichzusetzen sind, auch wenn viele Institutionen nicht sehr respektvoll mit ihren Klienten umgegangen sind. Die Rationalisierung (Zentralisierung und öffentliche Kontrolle) der Arbeitsvermittlung seit 1921, die sogenannten „Notstandsarbeiten“ und „produktive Erwerbslosenfürsorge" waren - in heutiger Ausdrucksweise - aktivierende, phasenweise stark genutzte Komponenten der Sozialpolitik der 1920er Jahre in Deutschland. Auch das unlängst ins SGB II überführte System der Sozialhilfe kannte immer wieder großangelegte Programme der „Hilfe zur Arbeit“ (Willing 2005b). Nicht zuletzt ist die der Aktivierung zugrundeliegende Idee der Hilfe zur Selbsthilfe tief in den Subtext des deutschen Wohlfahrtsstaates eingeschrieben und lässt sich problemlos zu den Unterstützungsvereinen der Arbeiterbewegung, den sozialpolitischen Doktrinen der katholischen Kirche im frühen 20. Jahrhundert und den ersten Armenordnungen der mittelalterlichen Städte zurückverfolgen.

In der Wirtschaftswunderära kam es zu einem schrittweisen Ausbau der Leistungstiefe und Inklusionsbreite der sozialen Sicherungssysteme (z. B. Frerich/Frey 1996b, S. 124f.; Trenk-Hinterberger 2006). Als mit dem Ende der Wirtschaftswunderära und ihrer Familien- und Sozialmodelle seit etwa 1974 (Lutz 1984) gleichzeitig Erwerbsbeteiligung und Arbeitslosenzahlen stiegen, reagierten die sozialstaatlichen Institutionen bald mit dem Ausbau der Hilfe-zurArbeit-Programme des BSHG und mit der Ausweitung der Arbeitsbeschaffungs- und Strukturanpassungsmaßnahmen des Arbeitsförderungsgesetzes (AFG) von 1969. Diese Programme waren zwar zunächst alle als Heranführung an reguläre Erwerbsarbeit gedacht, entwickelten sich jedoch mehr und mehr zu Ersatzarbeitsmärkten, deren rein arbeitsmarktpolitischer Nutzen zunehmend umstritten war (z. B. Hujer/ Thomsen 2006).

Auch hier folgen die neuen Maßnahmen und Instrumente des SGB II durchaus dem alten, von AFG und BSHG ausgetretenen Pfad. Das Beispiel der vielgepriesenen und vielkritisierten Ein-Euro-Jobs zeigt dies auch empirisch: Eine Bevorzugung von Problemgruppen am Arbeitsmarkt, für die das Instrument eigentlich gedacht war, lässt sich auf repräsentativer Basis nicht eindeutig feststellen. Die sogenannten „Einsperreffekte", 7 ihre Entdeckung bedeutete den arbeitsmarktpolitischen Tod des Vorgängerinstruments Arbeitsbeschaffungsmaßnahmen (ABM), fallen deutlich geringer aus. Westdeutsche Frauen, über 24-Jährige und Langzeitarbeitslose haben jedoch leichte Vorteile bei einer späteren Arbeitsplatzsuche (Bernhard et al. 2009, S. 186f.); Sprungbretteffekte durch direkte Übernahmen im Anschluss an den EinEuro-Job sind selten und vor allem dort zu beobachten, wo arbeitsmarktpolitische Maßnahmen eine Geschäftsgrundlage der jeweiligen Einrichtung sind. Erfolge scheinen sich hingegen vor allem in Dimensionen einzustellen, die im Zielsystem des SGB II nicht explizit genannt sind: Die kostengünstige Erbringung sozialer Dienstleistungen in einem eingespielten Trägernetzwerk (Klemm et al. 2009) und die Stabilisierung und Sozialintegration von Grundsicherungsempfängern - beides zwei zentrale Dimensionen der klassischen Förderarbeitsmärkte. Für Ersteres sprechen die starke Nachfrage von Trägern und Sozialeinrichtungen aller Art nach den Arbeitsgelegenheiten, die die vorher stark ausgedünnten ABM-Stellen ersetzen (Hohendanner et al. 2007), für Letzteres die ersten Ergebnisse einer breiten qualitativen Explorationsstudie (Promberger 2008b).

\section{Neue Akzente und Probleme in der Grundsicherung - ein Fazit}

Zunächst ist das SGB II wie seine Vorgänger schlichtweg ein wohlfahrtsstaatliches Regelwerk zur Bekämpfung von Armut, die auf gesellschaftliche, wirtschaftliche oder individuelle Ursachen zurückgeht. Den Geboten und Traditionen des Sozialstaats zufolge muss die Gesellschaft eingreifen, wenn einzelne Mitglieder in Not geraten und sich nicht eigenverantwortlich oder durch Hilfe aus ihrem Umfeld daraus befreien können. Ob das SGB II dies auch tatsächlich leistet, wo die Schwachstellen und Vorzüge des neuen Grundsicherungssystems liegen, ob und unter welchen Bedingungen Aktivierung funktioniert, bleibt trotz aller ersten Befunde eine offene Frage für Wissenschaft und Politik. Ein Bruch oder radikaler Wandel lässt sich jedoch dies sollte gezeigt werden - nicht erkennen. Langsamer Wandel auf bereits lange bestehenden Pfaden prägt das Bild. Folgende Tendenzen scheinen sich dabei auszuprägen:

\subsection{WANDLUNGSTENDENZEN}

In längerfristiger historischer Perspektive war der versorgende Wohlfahrtsstaat meist auch aktivierend, und auch der aktivierende Wohlfahrtsstaat muss immer auch dem Grundrecht seiner Bürger auf eine Unterstützung bei Hilfebedürftigkeit nachkommen. Dieses Grundrecht, das sich seit dem Grundsatzurteil des Bundesverwaltungsgerichts von 1954 mehr und mehr durchgesetzt hat, kann vielleicht als ein wichtiger, allmählich vollzogener Paradigmenwechsel in der Armutsbekämpfung gesehen werden, nach dem alle Bürger das Grundrecht auf Fürsorge durch die Gesellschaft besitzen - so hat T. H. Marshall (1992) dieses Prinzip eindrucksvoll formuliert. Dem stehen die Entbiografisierung und Entdifferenzierung der Hilfe gegenüber, gewissermaßen die Entkleidung der Betroffenen von statusbezogenen und biografischen Ansprüchen. Hier ist zum einen auf konfligierende Gerechtigkeitslogiken hinzuweisen: Der Logik der biografisch erworbenen Ansprüche auf Versorgung und Anerkennung als vollwertiger Arbeitsbürger, wie sie der Arbeitslosenhilfe zugrunde lag, steht die Logik der "letzten sozialen Solidarität" gegenüber, bei der der Hilfebedürftige erst aller biografischen und statusbezogenen Ansprüche - vom Restvermögen bis zum Anspruch auf Sozialversicherungsleistungen entkleidet sein muss, um in den Genuss der

\footnotetext{
7 "Einsperreffekte“ oder "Lock-In-Effekte" sind unbeabsichtigte Nebenwirkungen mancher arbeitsmarktpolitischer Maßnahmen. In der ökonomischen Literatur wird angenommen, dass die Maßnahme aufseiten der Teilnehmer zu Verhaltensweisen - etwa dem Rückgang von Jobsuchaktivitäten - führt, die die Betroffenen in der Arbeitslosigkeit festhalten, ,einsperren“.
} 
Grundsicherung zu kommen, wie wir es aus der Sozialhilfe kennen.

Zum anderen korrespondiert mit der Entdifferenzierung auch ein gewandeltes Bild vom erwerbsfähigen Hilfeempfänger: Es geht nicht mehr um den potenziell unmündigen Fürsorgeempfänger der Armutsbekämpfung vor 1960, nicht mehr um den Menschen, der darin unterstützt werden soll, in Würde zu leben und seine Eigenständigkeit wiederzuerlangen, wie es für weite Teile des Menschenbildes im BSHG 1961 kennzeichnend war. Stattdessen dominiert heute die Vorstellung des SGB II von einem vollständig dem Erwerbsparadigma subsumierten Arbeitsbürger, von dem nunmehr der Verhaltenskanon des „normalen“, eigenverantwortlich und rational handelnden, nachindustriellen bürgerlichen Arbeitnehmers erwartet wird.

\subsection{DAS KOMMODIFIZIERTE SUBJEKT}

Um es zuzuspitzen: Die volle Unterstützung des SGB II wird vor allem denjenigen zuerkannt, die für willens und in der Lage erachtet werden, sich in marktwirtschaftliche Verwertungszusammenhänge oder bei deren Fehlen - kompensatorische Einrichtungen einzufügen, somit auch bereit sind, Objekt in einem organisierten Kontext zu sein (Theuer/Sowa 2008). Der Kommodifizierungsbegriff ${ }^{8}$ drängt sich hier geradezu auf. Die Zwangsmaßnahmen des alten Fürsorgerechts (Arbeitszwang, Zwangseinweisung von „Auffälligen“ oder "Arbeitsscheuen") sind in diesem Modell zwar glücklicherweise vom Tisch, doch gleichzeitig wird von erwerbsfähigen Hilfebedürftigen im SGB II erwartet, dass sie über ein hohes Maß an Selbstkontrolle und Selbststeuerung (Zeitstrukturen im Alltag, Selbstpräsentation und Kommunikationskompetenz bei Behörden und Arbeitgebern), Flexibilität (Zumutbarkeitskriterien bei Jobangeboten), rationaler Lebensführung (z. B. Umgang mit pauschalierten geldförmigen Hilfeleistungen) und Konformität verfügen. Wo dies nicht der Fall ist oder zu sein scheint, drohen Sanktionen verschiedener Art - von der ungünstigen Auslegung der Vorschriften bis zu Kürzungen der Unterstützungsleistungen. Bei vielen erwerbsfähigen Hilfebedürftigen kann eine weitgehende Entsprechung zu diesem Subjektbild der Aktivierungspolitik vorausgesetzt werden. Doch dieses neue Paradigma vom aktivierbaren, weil rationalen und bürgerlich-normalen kommodifizierten Subjekt stößt erkennbar an Grenzen; und zwar nicht nur bei Hilfeempfängern mit kleinen Kindern, Krankheit oder Suchtproblemen. Grenzen zeigt auch die Frage auf, ob jemand, der gerade mal drei Stunden täglich arbeiten kann, auch tatsächlich Arbeit finden kann. Und wie sieht es aus bei physischen oder habituellen Abweichun- gen, abweichenden Handlungs- und Erwerbskompetenzen und schlechten, möglicherweise auch nur im wirtschaftlichen Wandel obsolet gewordenen Bildungs- und Qualifikationsvoraussetzungen? Normalitätsvorstellungen sind durchaus „normal“ für menschliche Gesellschaften und ihre Institutionen. Und so mag vielleicht für marktwirtschaftliche Arbeitsgesellschaften der kommodifizierte Arbeitsbürger „normal“ sein. Jedoch: Es gehört auch zum Grundbestand moderner, an den Menschenrechten orientierter Sozialstaatlichkeit, dass nicht alle Gesellschaftsmitglieder diesem Bild entsprechen und trotzdem in den Schutz des Sozialstaatsgebots fallen müssen. Dieser Tatsache muss sich auch die deutsche Aktivierungspolitik mit ihren veränderten normativen Anforderungen an die Hilfebedürftigen stellen, wenn sie sich nicht den subkutanen Fortbestand armenpolizeilicher Traditionen, einen Rückfall in den „punitiven Paternalismus" (Lessenich 2003; Offe 2001) oder die Rückgewinnung von sozialer Kontrolle durch Arbeit (Dahrendorf 2000) vorwerfen lassen will.

8 Kommodifizierung (Begriff von Polanyi 1978) meint hier insbesondere die Formung und Eingrenzung des Subjekts zum Anbieter der Ware Arbeitskraft (Marx 1984).

\section{LITERATUR}

Achatz, J./Dornette, J./Popp, S./Promberger, M./Schels, B./Wenzel, U./Wenzig, C./Wübbeke, C. (2009): Lebenszusammenhänge erwerbsfähiger Hilfebedürftiger im Kontext der Grundsicherungsreform, in: Möller, J./Walwei, U. (Hrsg.): Handbuch Arbeitsmarkt 2009, Nürnberg, S. 203-235

Bartelheimer, P. (2008): Wie man an seiner Eingliederung mitwirkt, in: Zeitschrift für Sozialreform (ZSR) 1, S. 11-37

Beck, U. (1999): Schöne neue Arbeitswelt, Frankfurt Becker, I./Hauser, R.(2005): Dunkelziffer der Armut, Berlin Bellmann, L./Gartner, H. (2003): Fakten zur Entwicklung der qualifikatorischen und sektoralen Lohnstruktur, in: Mitteilungen aus der Arbeitsmarkt- und Berufsforschung 4, S. 493-508

Bernhard, S./Hohmeyer, K./Jozwiak, E./Koch, S./Kruppe, T./Stephan, G./Wolff, J. (2009): Aktive Arbeitsmarktpolitik in Deutschland und ihre Wirkungen, in: Möller, J./Walwei, U. (Hrsg.): Handbuch Arbeitsmarkt 2009, Bielefeld, S. 149-201
Bosch, A. (2009): Ding und Symbol. Sozialstruktur, Identität und persönliche Objekte, Habilitationsschrift, Erlangen

Bundestag (2009): Endbericht - Evaluation der Experimentierklausel nach § 6c SGB II, Bundestagsdrucksache 16/11488

Castel, R. (2000): Die Metamorphosen der sozialen Frage, Konstanz Christoph, B. (2008): Was fehlt bei Hartz IV? Zum Lebensstandard der Empfänger von Leistungen nach SGB II, in: Informationsdienst Soziale Indikatoren, Juli, S. 7-10

Dahrendorf, R. (2000): Die globale Klasse und die neue Ungleichheit, in: Merkur 619, S. 1057-1068

Dingeldey, I. (2006): Aktivierender Wohlfahrtsstaat und sozialpolitische Steuerung, in: Aus Politik und Zeitgeschichte (APuZ) 8/9, S. 3-9

Dornette, J./Rauch, A./Schubert, M./Behrens, J./Höhne, A./Zimmermann, M. (2008): Auswirkungen der Einführung des SGB II auf erwerbsfähige hilfebedürftige Personen mit gesundheitlichen Beeinträchtigungen, in: Zeitschrift für Sozialreform (ZSR) 1, S. 79-96 
Ellguth, P./Kohaut, S. (2008): Tarifbindung und betriebliche Interessenvertretung, in: WSI-Mitteilungen 9, S. 515-519

Foucault, M. (1975): Überwachen und Strafen. Die Geburt des Gefängnisses, Frankfurt/M.

Frerich, J./Frey, M. (1996a,b): Handbuch der Geschichte der Sozialpolitik, Band 1(1996a) und Band 3 (1996b), München/Wien

Friedeburg, R. v. (2002): Lebenswelt und Kultur der unterständischen Schichten in der frühen Neuzeit. Enzyklopädie deutscher Geschichte, Bd. 62, München

Gebauer, R./Petschauer, H./Vobruba, G. (2002): Wer sitzt in der Armutsfalle?, Berlin

Hirseland, A./Ramos-Lobato, P./Promberger, M. (2009): Teilhabeprobleme von erwerbsfähigen Hilfebedürftigen, Ms., Nürnberg (im Erscheinen) Hohendanner, Ch./Promberger, M./Bellmann, L. (2007): Ein-Euro-Jobs in deutschen Betrieben. Mehr als "alter Wein in neuen Schläuchen"?, in: Sozialer Fortschritt 12, S. 300-309

Hujer, R./Thomsen, S. (2006): Wirksamkeit von Arbeitsbeschaffungsmaßnahmen in Deutschland. Empirische Befunde mikroökonometrischer Analysen, in: Zeitschrift für ArbeitsmarktForschung (ZAF) 3/4, S. 329345

Hradil, S. (2006): Gibt es in Deutschland eine Unterschicht?, in: Gesellschaft - Wirtschaft - Politik (GWP) 4, S. 440-444

Koch, S./Kupka, P./Steinke, J. (2009): Aktivierung, Erwerbstätigkeit und Teilhabe, Nürnberg

Klemm, M./Sowa, F./Hohendanner, Ch./Promberger, M. (2009): HartzIV-Reform, Arbeitsgelegenheiten im "Netz der Akteure", in: Neue Praxis 1, S. 75-85

Kronauer, M. (2002): Exklusion, Frankfurt/New York

Lessenich, S. (2003): Der Arme in der Aktivgesellschaft - zum sozialen Sinn des "Förderns und Forderns", in: WSI-Mitteilungen 4, S. 214-220

Lis, C./Soly, H. (1979): Poverty and Capitalism in pre-industrial Europe, Bristol

Ludwig-Mayerhofer, W./Behrend, O./Sondermann, A. (2009): Auf der Suche nach der verlorenen Arbeit. Arbeitslose und Arbeitsvermittler im neuen Arbeitsmarktregime, Konstanz

Lutz, B. (1984): Der kurze Traum immerwährender Prosperität, Frankfurt Marshall, T. H. (1992): Bürgerrechte und soziale Klassen. Zur Soziologie des Wohlfahrtsstaates, Frankfurt (1981)

Marx, K. (1984): Das Kapital, Bd. 1, Der Produktionsprozess des Kapitals, Berlin (1867)

Offe, C. (2001): Wessen Wohl ist das Gemeinwohl?, in: Wingert, L./ Günther, K. (Hrsg.): Die Öffentlichkeit der Vernunft und die Vernunft der Öffentlichkeit, Frankfurt/M., S. 459-488

Polanyi, K. (1978): The Great Transformation, Frankfurt
Prell, M. (1997): Sozialökonomische Untersuchungen zur Armut im antiken Rom, Stuttgart

Promberger, M. (Hrsg.) (2007): Neue Daten für die Sozialstaatsforschung, IAB-Forschungsbericht 12, Nürnberg

Promberger, M. (2008a): Hartz IV - Neue Angebote gefragt, in: SOZIALwirtschaft 1, S. 6-9

Promberger, M. (2008b): Arbeit, Arbeitslosigkeit und soziale Integration, in: Aus Politik und Zeitgeschichte (APuZ) 40/41, S. 7-15

Rudloff, W. (1998): Öffentliche Fürsorge, in: Hockertz, H.-G. (Hrsg.): Drei Wege deutscher Sozialstaatlichkeit. NS-Diktatur, Bundesrepublik und DDR im Vergleich, München, S. 191-229

Rudolph, H. (2006): Entwicklung der Anzahl der Leistungsempfänger in der Grundsicherung für Arbeitssuchende, in: Bundesagentur für Arbeit, Nürnberg (Hrsg.): SGB II, Sozialgesetzbuch Zweites Buch. Grundsicherung für Arbeitssuchende. Zahlen, Daten, Fakten. Jahresbericht 2005, Nürnberg, S. 11-14

Sachße, Ch./Tennstedt, F. (1980): Geschichte der Armenfürsorge in Deutschland. Bd. 1.: Vom Spätmittelalter bis zum 1. Weltkrieg, Stuttgart Schlemmer, Th./Raithel, Th. (2009): Die Rückkehr der Arbeitslosigkeit. Die Bundesrepublik Deutschland im europäischen Kontext. Zeitgeschichte im Gespräch, Bd. 5, herausg. v. Institut für Zeitgeschichte, München (im Erscheinen)

Schmid, G./Oschmiansky, F. (2005): Arbeitsmarktpolitik/Arbeitslosenversicherung, in: Schmidt, M. G. (Hrsg.): Bundesrepublik Deutschland 19821989. Finanzielle Konsolidierung und institutionelle Reform. Geschichte der Sozialpolitik in Deutschland seit 1945, Bd. 7, S. 238-289

Theuer, S./Sowa, F. (2010): Vom Subjekt zum Objekt? Die Reform der öffentlichen Arbeitsverwaltung und ihre Konsequenzen für Arbeitslose in Deutschland. Vortragsmanuskript, Tagungsband des 34. Kongresses der Deutschen Gesellschaft für Soziologie (DGS) in Jena (im Erscheinen) Thießen, F./Fischer, C. (2008): Die Höhe der sozialen Mindestsicherung. Eine Neuberechnung „bottom up“, in: Zeitschrift für Wirtschaftspolitik 2, S. $145-173$

Trenk-Hinterberger, P. (2006): Sozialhilfe, in: Hockerts, H.-G. (Hrsg.): Bundesrepublik Deutschland 1966-1974. Eine Zeit vielfältigen Aufbruchs. Geschichte der Sozialpolitik in Deutschland seit 1945, Bd. 5, S. 593-631 Wenzel, U. (2008): Fördern und Fordern aus Sicht der Betroffenen, in: Zeitschrift für Sozialreform 1, S. 57-78

Willing, M. (2005a): Fürsorge, in: Schulz, G. (Hrsg.): Bewältigung der Kriegsfolgen und Rückkehr zur sozialpolitischen Normalität, Geschichte der Sozialpolitik in Deutschland seit 1945, Bd. 3, S. 559-596 Willing, M. (2005b): Sozialhilfe, in: Schmidt, M. G. (Hrsg.): Bundesrepublik Deutschland 1982-1989. Finanzielle Konsolidierung und institutionelle Reform. Geschichte der Sozialpolitik in Deutschland seit 1945, Bd. 7, S. 480-516 